
Pesquisa

DEI

DEPARTAMENTO

DE ENGENHARIA

INDUSTRIAL

Número 05 | 052021

\title{
Avaliação de Usinas Híbridas por meio da Teoria de Opções Reais
}

$$
\text { Autor(es): }
$$

Diego Nascimento Maia 


\title{
Série dos Seminários de Acompanhamento à Pesquisa
}

\section{Avaliação de Usinas Híbridas por meio da Teoria de Opções Reais}

\author{
Autor(es): \\ Diego Nascimento Maia
}

CRÉDITOS:

SISTEMA MAXWELL / LAMBDA

https://www.maxwell.vrac.puc-rio.br/

Organizadores: Fernanda Baião / Soraida Aguilar 


\section{AGENDA}

> Apresentação pessoal

$>$ Objetivo

> Contextualização

$>$ Metodologia

$>$ Resultados Preliminares

> Referências 


\section{APRESENTAÇÃO PESSOAL}

\section{DIEGO NASCIMENTO MAIA}

$>$ Engenheiro De Computação (IME)

> Pesquisador desde 2015 no Centro de Pesquisas em Energia Elétrica (CEPEL)

$>\quad$ Início do Mestrado: 2018.2

$>$ Orientador: Luiz Eduardo Brandão

$>$ Co-orientador: Fábio Rodrigo Batista

> Área de Concentração e Linha de Pesquisa: Finanças e Análise de Investimentos / Finanças Corporativas 


\section{OBJETIVO}

> Determinar o melhor modelo de negócios para um agente gerador de energia elétrica no Brasil, considerando as seguintes possibilidades:

$\checkmark \quad$ Investir em uma usina de uma tecnologia

- Eólica

- Solar

$\checkmark \quad$ Investir em um arranjo híbrido

- Eólica + Solar

- Eólica + Solar + Bateria 


\section{CONTEXTUALIZAÇÃO}

> Análise Econômico-Financeira de Projetos

Fluxos de Caixa

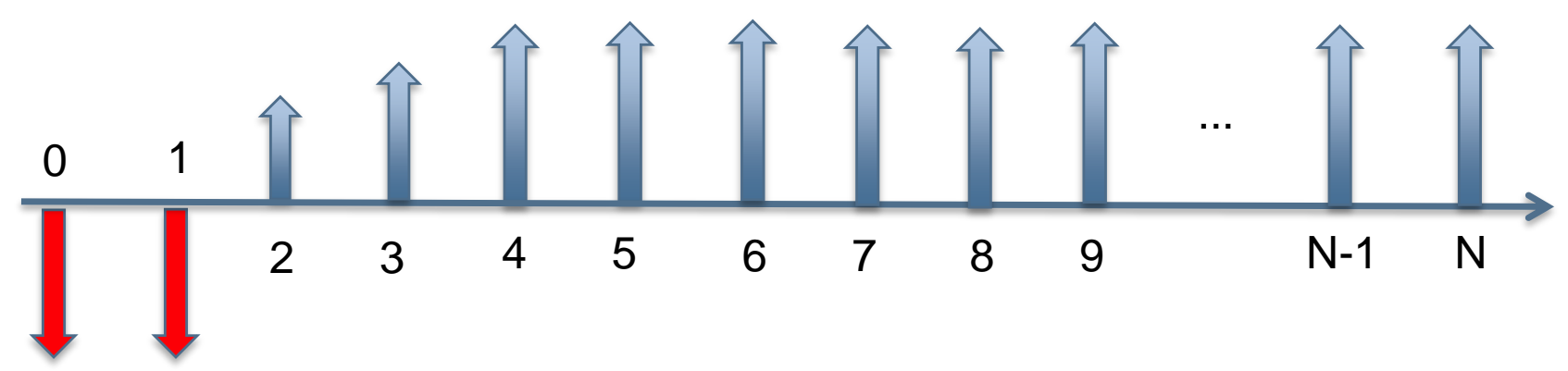

Investimentos

Para Projetos de Geração de Energia

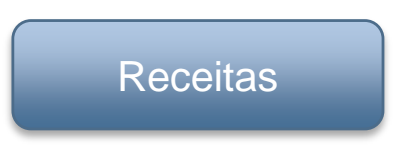

Receita com Energia Gerada

Receita no MCP

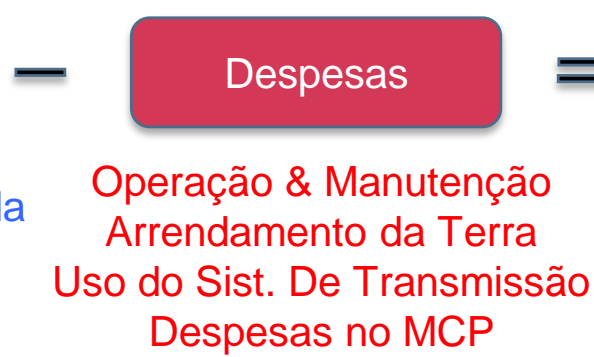

INDICADORES

DE

VIABILIDADE 


\section{CONTEXTUALIZAÇÃO}

> Geração Eólica e Solar estão entre as fontes de geração que mais crescem na matriz energética brasileira
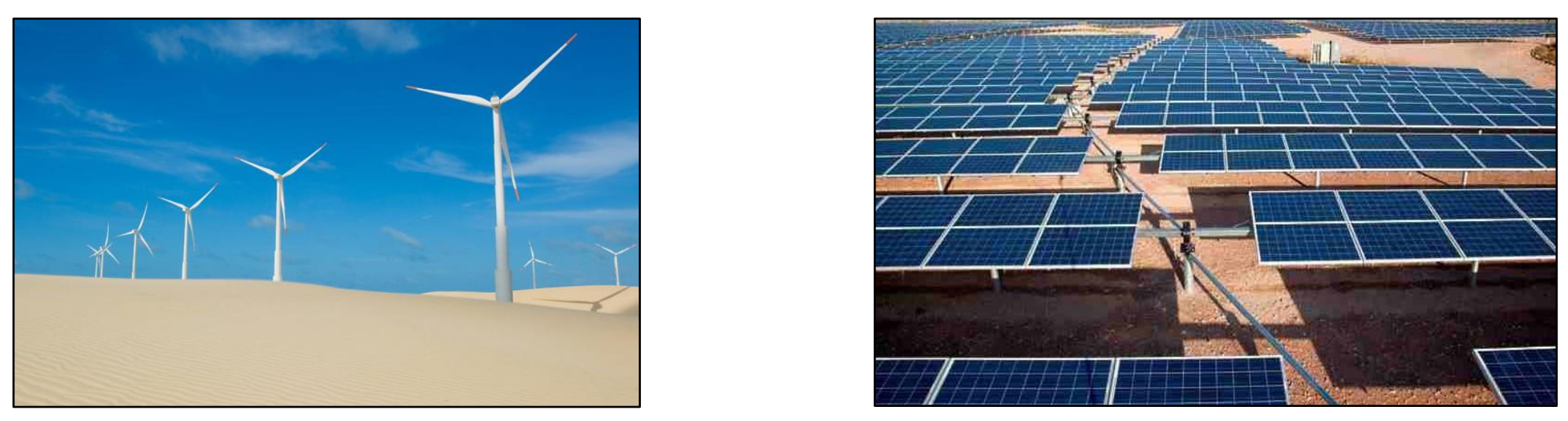


\section{CONTEXTUALIZAÇÃO}
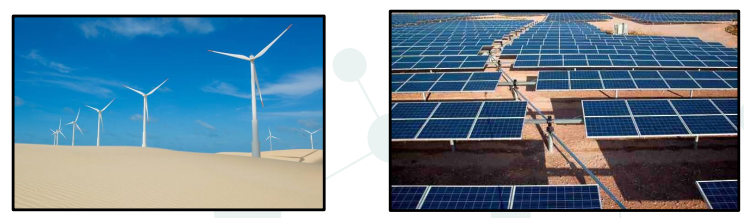

> Geração Eólica e Solar estão entre as fontes de geração que mais crescem na matriz energética brasileira

\begin{tabular}{|c|c|c|c|c|c|c|c|c|c|c|c|}
\hline SONTF(a) & 2019 & 2020 & 2021 & 2022 & 2023 & 2024 & 2025 & 2026 & 2027 & 2028 & 2029 \\
\hline RENOVÁVEIS & 138.284 & 140.002 & 141.026 & 142.036 & 148.366 & 154.196 & 158.776 & 163.741 & 168.739 & 173.814 & 178.915 \\
\hline$H I D R O^{(b)}$ & 101.288 & 101.899 & 101.935 & 101.935 & 102.139 & 102.139 & 102.139 & 102.523 & 102.942 & 103.436 & 103.958 \\
\hline OUTRAS RENOVÁVEIS: & 36.996 & 38.103 & 39.091 & 40.101 & 46.227 & 52.057 & 56.637 & 61.217 & 65.797 & 70.377 & 74.957 \\
\hline $\mathrm{PCH}$ e CGH & 6.385 & 6.610 & 6.787 & 6.898 & 7.207 & 7.545 & 7.845 & 8.145 & 8.445 & 8.745 & 9.045163 \\
\hline EÓLICA & 15.017 & 15.370 & 15.477 & 15.742 & 20.263 & 24.475 & 27.475 & 30.475 & 33.475 & 36.475 & 39.475 \\
\hline BIOMASSA $^{(a)}+$ BIOGÁS & 13.412 & 13.643 & 13.790 & 13.840 & 14.135 & 14.415 & 14.695 & 14.975 & 15.255 & 15.535 & 13.015 \\
\hline SOLAR CENTRALIZADA & 2.182 & 2.480 & 3.037 & 3.622 & 4.622 & 5.622 & 6.622 & 7.622 & 8.622 & 9.622 & 10.622 \\
\hline NÃO RENOVÁVEIS & 22.672 & 24.474 & 25.779 & 25.779 & 27.705 & 29.293 & 29.239 & 33.114 & 35.319 & 36.916 & 42.059 \\
\hline URÂNIO & 1.990 & 1.990 & 1.990 & 1.990 & 1.990 & 1.990 & 1.990 & 3.395 & 3.395 & 3.395 & 3.395387 \\
\hline GÁS NATURAL $L^{(t)}$ & 12.921 & 14.436 & 15.741 & 15.741 & 17.859 & 21.234 & 22.662 & 25.679 & 28.315 & 31.339 & 36.190 \\
\hline CARVÃO & 3.017 & 3.017 & 3.017 & 3.017 & 3.017 & 3.017 & 3.017 & 3.017 & 3.017 & 1.790 & 2.083 \\
\hline ÓLEO COMBUSTÍVEL'(e) & 3.697 & 3.697 & 3.697 & 3.697 & 3.697 & 2.510 & 1.028 & 657 & 226 & 25 & 25 \\
\hline ÓLEO DIESEL & 1.047 & 1.333 & 1.333 & 1.333 & 1.141 & 542 & 542 & 366 & 366 & 366 & 366 \\
\hline TOTALDOSIN & 160.956 & 164.476 & 166.805 & 167.814 & 176.070 & 183.489 & 188.015 & 196.855 & 204.059 & 210.729 & 220.974 \\
\hline Fonte: PDE 2029 & & & & & & & & & & & $37 \%$ \\
\hline
\end{tabular}




\section{CONTEXTUALIZAÇÃO}

> Conceito de Usina Híbrida

$\checkmark$ "Uma instalação de geração de energia que converte energia primária em energia elétrica e que consiste em mais de um módulo de geração de energia conectado a uma rede em um ponto de conexão."
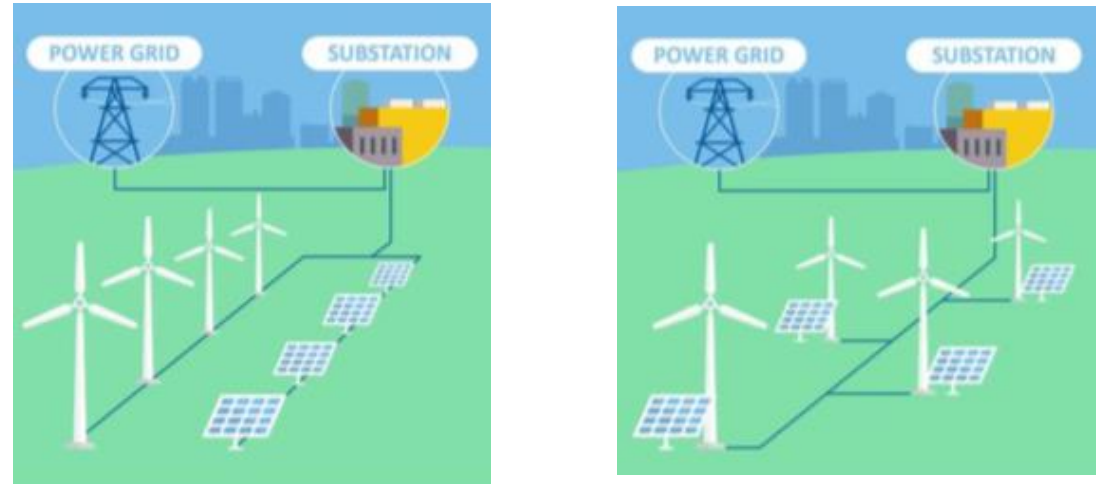

Fonte: WindEurope 


\section{CONTEXTUALIZAÇÃO}

> Geração Eólica e Solar estão entre as fontes de geração que mais crescem na matriz energética brasileira

$\checkmark$ Hibridização pode aumentar a atratividade de projetos anteriormente inviáveis

$\checkmark$ Complementaridade na geração dessas fontes

- Geração Solar ocorre no período diurno

- Por exemplo, em localidades no Nordeste a geração eólica ocorre com maior intensidade no período noturno

- Utilização mais eficiente da rede elétrica

$\checkmark$ Regulamentação necessária

- Em discussão desde 2017

- Consulta pública em 2019

- Agenda Regulatória ANEEL 2020 - 2021 


\section{CONTEXTUALIZAÇÃO}

> Projeto Híbrido Eólica + Solar

$\checkmark$ Ganhos sinérgicos de Custos na Construção e na Operação da Usina

$\checkmark$ Curtailment

$\checkmark \quad$ Efeito Portfólio

$>$ Armazenamento em Baterias

$\checkmark$ Aproveitamento da diferenças de preços durante um dia

$\checkmark$ Redução de custo da tecnologia

$\checkmark$ Redução do Curtailment 


\section{RISCOS ENVOLVIDOS}

Pontes de Geração

$\checkmark$ Geração Eólica: Vento

$\checkmark$ Geração Solar: Irradiância Solar

$\checkmark$ Complementaridade entre Eólica e Solar

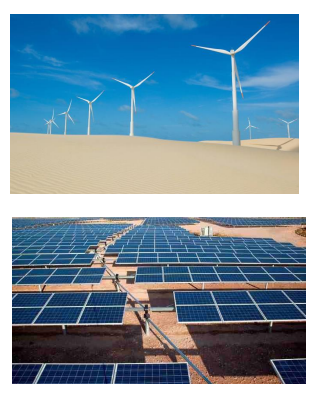

> Preço da Energia no Mercado de Curto Prazo

$$
\begin{gathered}
\text { PLD } \\
\text { PREÇO DE } \\
\text { LIQUIDAÇÃO DAS } \\
\text { DIFERENÇAS }
\end{gathered}
$$




\section{RISCOS ENVOLVIDOS}

\section{> Preço da Energia no Mercado de Curto Prazo}

$\checkmark \quad$ Atualmente: Preço por semana por Submercado

$\checkmark$ A partir de 2021: Preço por hora por Submercado

- Modelo tem previsão de 1 semana

- Como fica análise de 30 anos do projeto híbrido? 


\section{RISCOS ENVOLVIDOS}

> Tratamento relativo ao PLD Horário
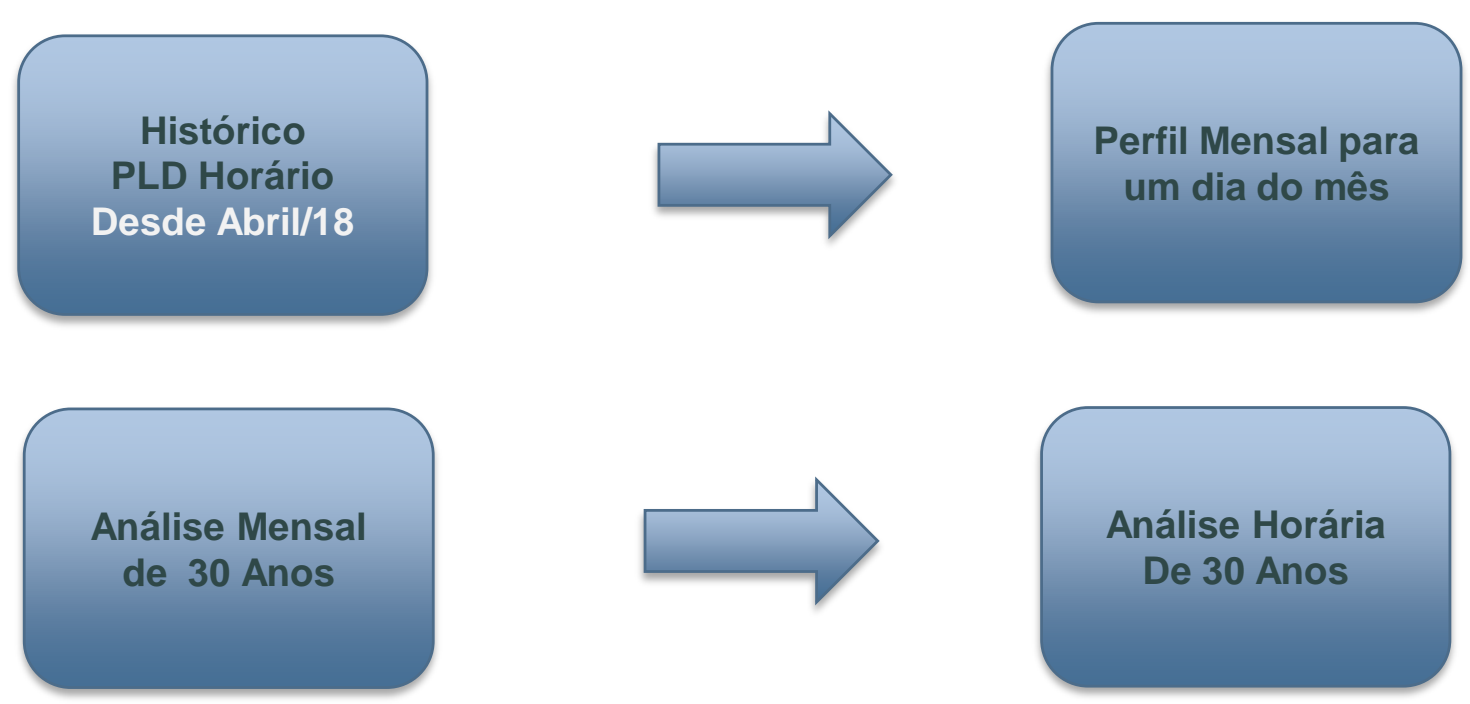

$\checkmark \quad$ O que muda com PLD Horário?

- Exposição ao PLD durante o dia 


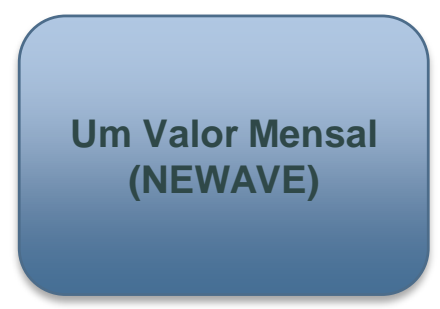

Fatores de

Desagregação

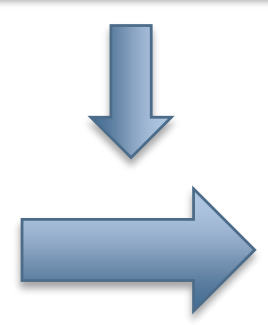

24 valores

(Um para cada hora do dia) 


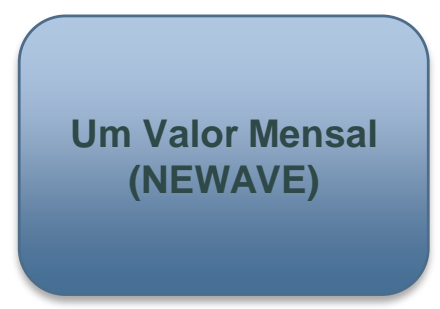

Fatores de

Desagregação

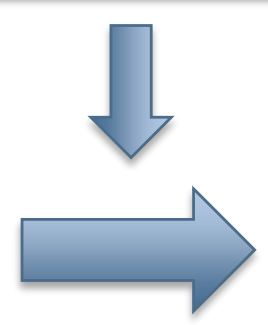

24 valores

(Um para cada hora do dia) 


\section{TEORIA DAS OPÇÕES REAIS}

$>$ Opção Financeira

$\checkmark$ Instrumento financeiro que atribui ao comprador o direito de comprar ou vender determinado ativo numa data ou até uma data

$>$ Opção Real

$\checkmark$ Ativos Reais (tangíveis) em vez de ativos financeiros

$\checkmark$ Exemplo do cotidiano: Passagem Aérea Reembolsável

$\checkmark$ Exemplo em avaliação de projetos: Adiar investimento em um projeto

$>$ Princípios da Teoria

$\checkmark$ Flexibilidade Gerencial

$\checkmark$ Incerteza

$\checkmark$ Irreversibilidade 


\section{TEORIA DAS OPÇÕES REAIS}

> Teoria das Opções Reais aplicado às Usinas Híbridas
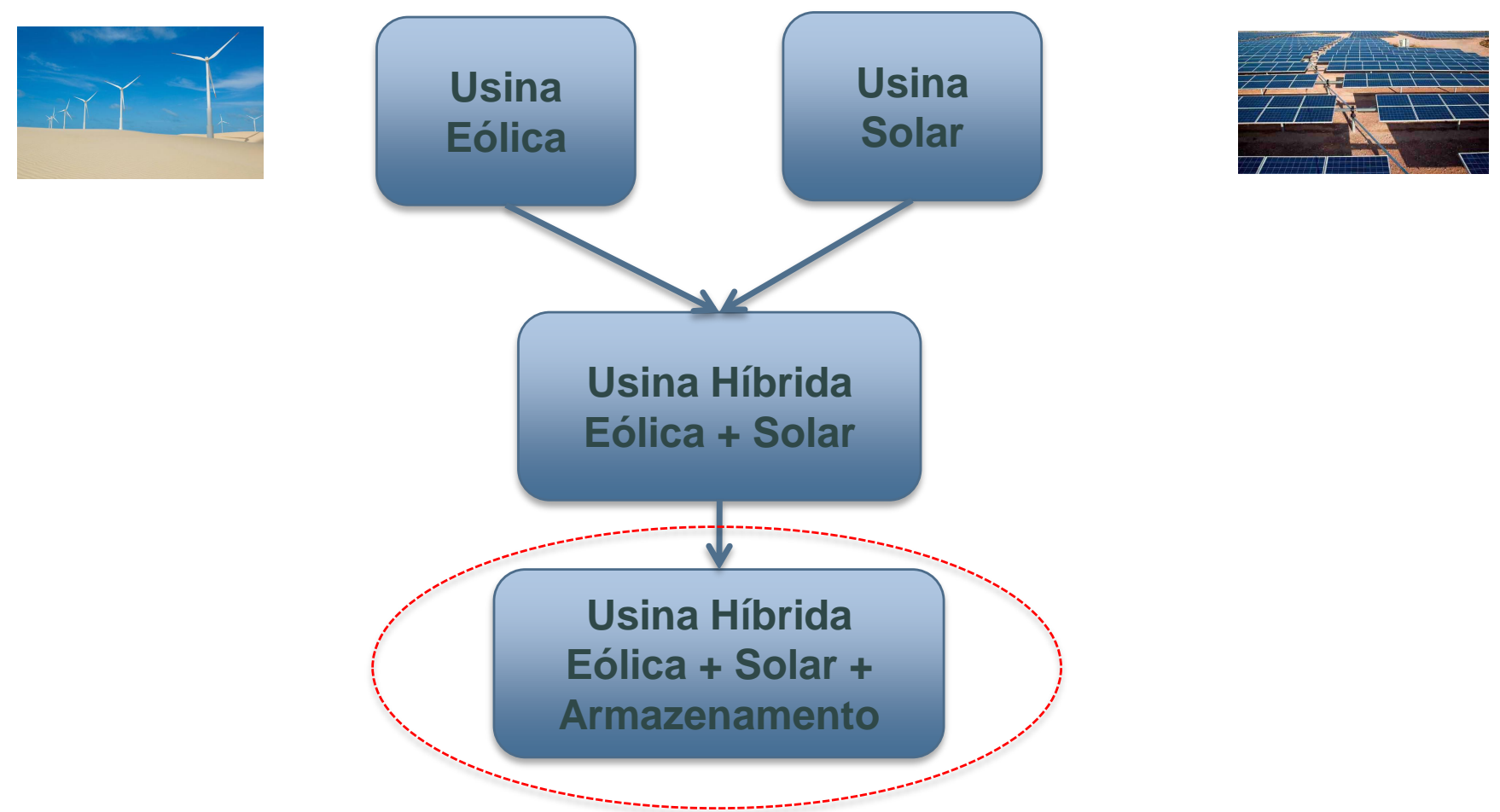


\section{METODOLOGIA}

Análise determinística individual dos empreendimentos

Análise determinística do arranjo híbrido dos empreendimentos

Análise de dados das séries históricas de geração eólica e solar por meio de reanálise

Análise de Risco dos fatores de risco nas análises individuais e híbrida

Análise do arranjo híbrido com o uso de baterias de armazenamento 


\section{RESULTADOS PRELIMINARES}

> Boxplots para cada hora do dia no mês

$\checkmark$ Exemplo: Janeiro
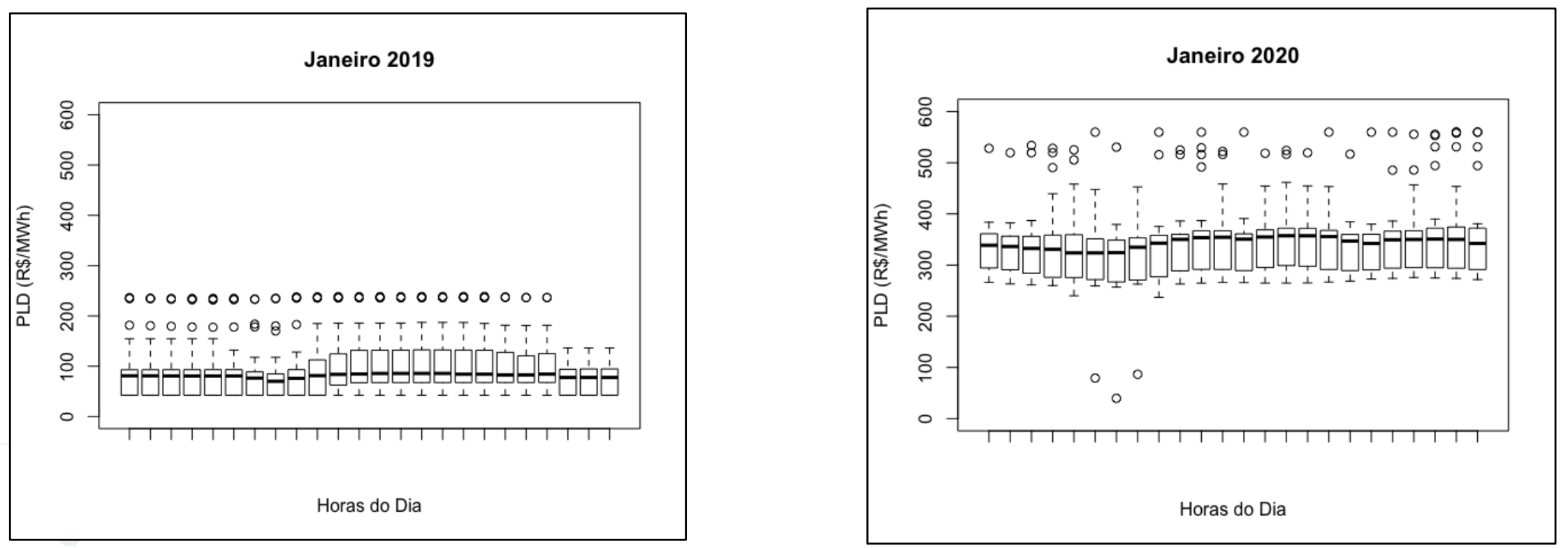


\section{REFERÊNCIAS}

> ZAMBELI, M.S., FARIA, J. M, SOUZA, A.A., Metodologia Para Análise De Risco Com PLD Horário, XXV Seminário Nacional de Produção e Transmissão de Energia Elétrica, Belo Horizonte, Brasil, 2019.

> MME, EPE. Plano Decenal de Expansão de Energia 2029, Brasília, Brasil, 2019.

> WINDEUROPE. Renewable Hybrid Power Plants: Exploring the Benefits and Market Opportunities, Brussels, Belgium, 2019.

$>$ KLONARI, V., Exploring the Viability of Hybrid Wind-Solar Power Plants, 4th International Hybrid Power Systems Workshop, Crete, Greece, 2019.

> DAS, K., Enhanced Features of Wind-Based Hybrid Power Plants, 4th International Hybrid Power Systems Workshop, Crete, Greece, 2019. 
OBRIGADO!

DIEGO.N.MAIA@GMAIL.COM 


\section{METODOLOGIA}

$>$ Análise do Arranjo Híbrido com o uso de Baterias

$>$ Especificação da bateria;

> Avaliação da opção de se investir no uso de baterias para o arranjo híbrido estudado
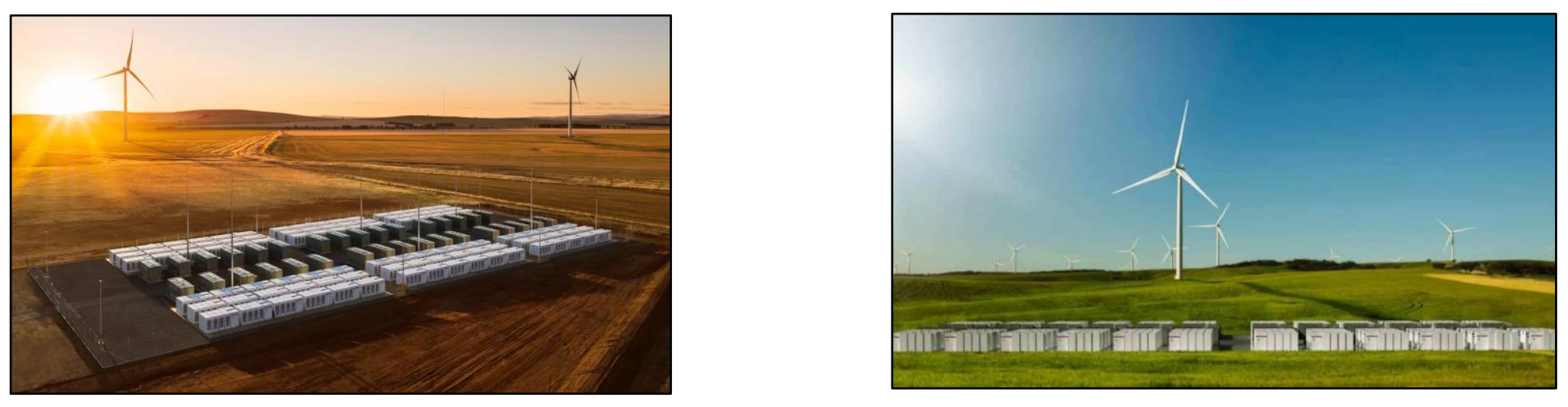


\section{METODOLOGIA}

\section{> Análise determinística individual dos empreendimentos}

> Especificação das características técnicas de uma usina eólica, e outra solar fotovoltaica, separadamente

> Obtenção de série histórica de geração, em base mensal, para cada usina isoladamente;

> Geração de diferentes cenários de produção energética, em base mensal, para cada usina separadamente;

> Obtenção de diferentes cenários de PLDs em base mensal;

> Realização de uma análise de risco de cada projeto separadamente 


\section{METODOLOGIA}

\section{> Análise determinística do arranjo híbrido dos empreendimentos}

> Determinação de quais características individuais seriam alteradas em função do arranjo híbrido e posterior especificação da usina

$>$ Em base mensal, realização de uma análise de risco do arranjo híbrido;

> Levantamento de perfil típico da geração eólica e solar fotovoltaica, em base horária, no local de desenvolvimento da usina;

> Levantamento de perfil típico do PLD horário no submercado de desenvolvimento da usina;

> Obtenção de cenários de PLDs e geração em base horária;

> Realização de uma análise de risco da usina híbrida em base horária. 


\section{CONTEXTUALIZAÇÃO}

> Regulamentação necessária

\section{HOJE}
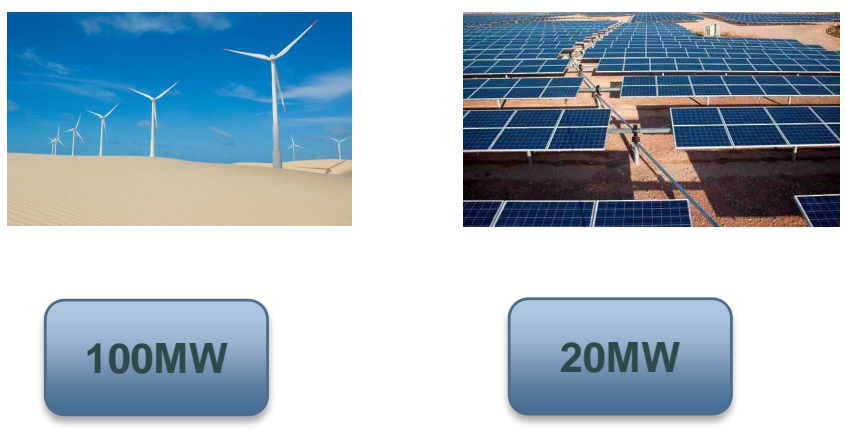

20MW

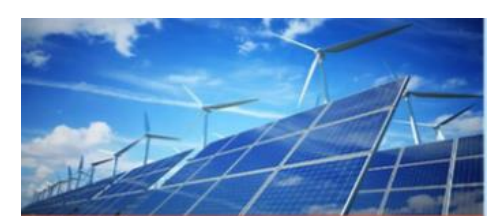

$120 \mathrm{MW}$

\section{EM ESTUDO}
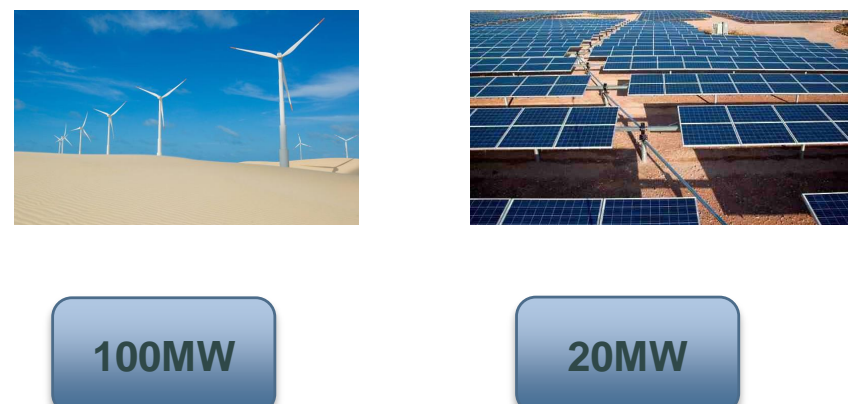

20MW

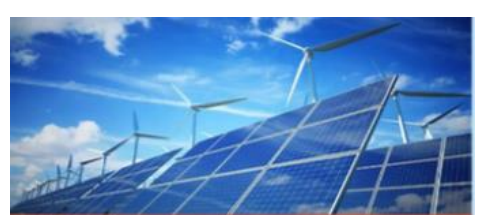

